\title{
Complete and incomplete revascularization: Whose definition is it anyway?
}

\author{
Shreenidhi Venuraju, MBBS, MRCP, a and Avijit Lahiri, MBBS, MRCP, MSc, \\ FACC $^{\mathrm{a}, \mathrm{b}, \mathrm{c}}$ \\ ${ }^{a}$ Clinical Imaging and Research Centre, Wellington Hospital, London, UK \\ ${ }^{b}$ Imperial College, London, UK \\ ${ }^{\mathrm{c}}$ Middlesex University, London, UK
}

Received Apr 6, 2015; accepted Apr 17, 2015

doi: 10.1007/s12350-015-0166-8

\section{See related article, pp. 546-555}

Management of significant coronary artery disease is comprised of medical management, whereby risk factors are addressed, and coronary revascularization. The data regarding the survival benefits afforded by emergent coronary revascularization of the culprit vessel in ST elevation myocardial infarction (STEMI) are robust and irrefutable. ${ }^{1}$ Revascularization in stable patients with chest pain and the factors guiding it is certainly more contentious. Boden et $\mathrm{al}^{2}{ }^{2}$ in their landmark, COURAGE trial, showed that despite objective evidence of ischaemia, revascularization with bare metal stents in the patients of the per-cutaneous coronary intervention (PCI) plus optimal medical therapy arm did not provide any survival benefit when compared to optimal medical therapy alone. It is also important to note that target revascularization was always attempted and complete revascularization was undertaken where clinically appropriate in the PCI arm. They concluded that revascularization could be delayed without adverse outcomes in this group of patients with stable heart disease even with evidence of ischaemia in the medium term.

Does this imply that revascularization should be the last resort in patients with refractory angina or the sole preserve of acute coronary syndromes? Even when the decision has been made to proceed with revascularization, there are a multitude of questions that need

Reprint requests: Shreenidhi Venuraju, Clinical Imaging and Research Centre, Wellington Hospital, St. Johns Wood, London, NW89LE, UK; shreenidhimv@gmail.com.

J Nucl Cardiol 2016;23:556-9.

1071-3581/\$34.00

Copyright (C) 2015 American Society of Nuclear Cardiology. answered. Do we target just the culprit lesion or is there a role for complete revascularization, whereby all plaques deemed obstructive on invasive coronary angiography are stented? Some may argue that it would be most prudent to let ischaemia guide the revascularization. Physiologically, this argument is unimpeachable but its application would hinge on a functional test that provided evidence of not just degree of stenosis in a single vessel but also multi-vessel disease with a high degree of sensitivity and specificity. The largest body of evidence belongs to myocardial perfusion imaging (MPI) and its value, not just in the diagnosis of ischaemic heart disease but also in predicting short- and medium-term prognosis is well established. ${ }^{3}$

In the paper by Li et al in this issue of the Journal of Nuclear Cardiology, the authors have conducted a retrospective study of 170 patients where in they have explored complete vs incomplete coronary revascularization (CCR vs ICR) guided either by coronary angiography (CA) parameters or by myocardial ischaemic burden using MPI. Patients were followed up for $47+21$ months and evaluated for a primary end point of all-cause mortality and secondary end points of all major adverse cardiac events and repeat revascularization. For the sake of analysis, four sub-groups were created, CCR-CA + CCR-MPI (Group 1), CCR-CA + ICR-MPI (Group 2), ICR-CA + CCR-MPI (Group 3), and ICR-CA and ICR-MPI (Group 4). All-cause mortality and MACE were significantly different in patients who underwent CCR as compared to those with ICR based on MPI parameters. When CCR and ICR were compared using the CA criteria, there was a difference noted but it was not statistically significant. Cumulative incidence of mortality and MACE was also significantly different in patients undergoing CCR by both CA and MPI parameters when compared to those who had incomplete revascularization based on both CA and MPI 
criteria. It is worth noting that patients in Group 4 had significantly more severe and extensive disease, which could be a major confounding factor. Although no numbers have been quoted, ICR, in majority of the cases appears to be due to technical difficulty rather than intentional. Despite these limitations and small numbers, this study adds some valuable information to the existing body of knowledge, particularly since both criteria were evaluated in the same patient population. Another fact highlighted by this study is the glaringly obvious mismatch between coronary anatomical information and myocardial ischaemia; this mismatch occurred in nearly $50 \%$ of patients.

Stable coronary artery disease (CAD) typically presents with angina and maybe accompanied by dyspnoea. The severity of symptoms cannot be relied upon to facilitate management decisions as it does not reflect the severity of stenosis. Classifying CAD as single vessel, double vessel, or triple vessel disease based on coronary angiography data is the simplest way of describing the extent and severity of CAD. This classification also provides significant prognostic data aiding the clinical decision making process. The decision to opt for intensive medical therapy or to revascularize either with PCI or coronary artery bypass graft surgery can be made based on this information. Myocardial revascularization has to be undertaken after due consideration of the benefits of the procedure in terms of either survival or improvement in quality of life due to relief of symptoms that were significantly limiting patient activity. Apart from the simple description mentioned above, there are various scores that have been developed over the years that help predict major adverse cardiac events (MACE). For PCI, SYNTAX score has been validated and has proven to be an independent predictor of MACE. ${ }^{4}$ One of the major recommendations of the ESC guidelines on revascularization in 2010 was to dissociate it from diagnostic angiography. ${ }^{5}$ This would then allow a Heart Team comprising of a clinical cardiologist, cardio-thoracic surgeon, and an interventional cardiologist to deliberate the merits of the various management choices. There has been much debate over these recommendations, as many would argue for the provision of ad hoc PCI at the time of angiography particularly if the coronary anatomy was suitable, thereby avoiding a second invasive procedure the requirement of which itself would itself signify a higher risk patient.

The COURAGE data appear to suggest the noninferiority of optimal medical therapy compared to PCI but there were significant limitations that were not immediately apparent in the face of the headline grabbing conclusions. The inclusion of patients with mild ischaemia was one of the biggest limitations of the study.
Interestingly, after 4.6 years of follow-up, $21 \%$ in the PCI group underwent repeat revascularization, whereas $32.6 \%$ did so in the optimal medical therapy (OMT) arm of the study (HR:0.6; 95\% CI 0.51-0.71; $P<0.001$ ). In essence, one third of the patients in the OMT arm ended up having some form of revascularization during followup. A large meta-analysis of 28 studies involving 13,121 patients reported lower mortality rates in patients revascularized with PCI as compared to optimal medical therapy alone with an OR of 0.82 (95\% CI 0.68-0.99). ${ }^{6}$ It is noteworthy that the vast majority of patients in the PTCA studies were treated with either balloon angioplasty or bare metal stent implantation. It has been shown subsequently that the rates of re-stenosis with the newer drug eluting stents is far lesser compared to either of the older strategies thus it can be expected that the size of this effect could be greater in a future analysis. This meta-analysis is significant as studies that compared revascularization and medical therapy were underpowered to detect significant differences in mortality in the two arms. What becomes clear is that it is not intervention vs drug therapy, it is optimal use of both based on patient need. Intensive medical therapy is the first step in management of stable angina. All patients with diagnosis of stable CAD should be on Aspirin, HMGCoA Reductase inhibitor (statin), and a betablocker along with glyceryl tri-nitrate spray to provide symptomatic relief. Addition of long-acting nitrates, Ivabradine, or Ranolazine is based on severity of symptoms and the limitation imposed on daily activity by angina. There are a couple of instances when revascularization is the logical next step in management based on anatomical data alone even in the absence of any symptoms or without any functional assessment of ischaemic burden: left main stem stenosis of $>50 \%$ and multi-vessel disease with left ventricular systolic dysfunction.

In the absence of a coronary anatomy-induced imperative, what drives the decision to revascularize? In the Asymptomatic Cardiac Ischaemia Pilot (ACIP) study, ${ }^{7}$ patients with coronary anatomy suitable for revascularization were randomized into angina-guided drug therapy, angina- and ischaemia-guided drug therapy, and revascularization with per-cutaneous transluminal angioplasty (PTCA) or coronary artery bypass graft surgery $(\mathrm{CABG})$. At two years the cumulative mortality rates were significantly higher in the angina and ischaemia guided medical management arms $(6.6 \%$ and $4.4 \%$ respectively) when compared to the revascularization arm (1.1\%) $(\mathrm{p}<0.005$ and $\mathrm{p}=0.05$ respectively). There was no difference between the angina-guided and ischaemia-guided strategies. The next question that then arises is what degree of ischaemic burden should warrant a referral for 


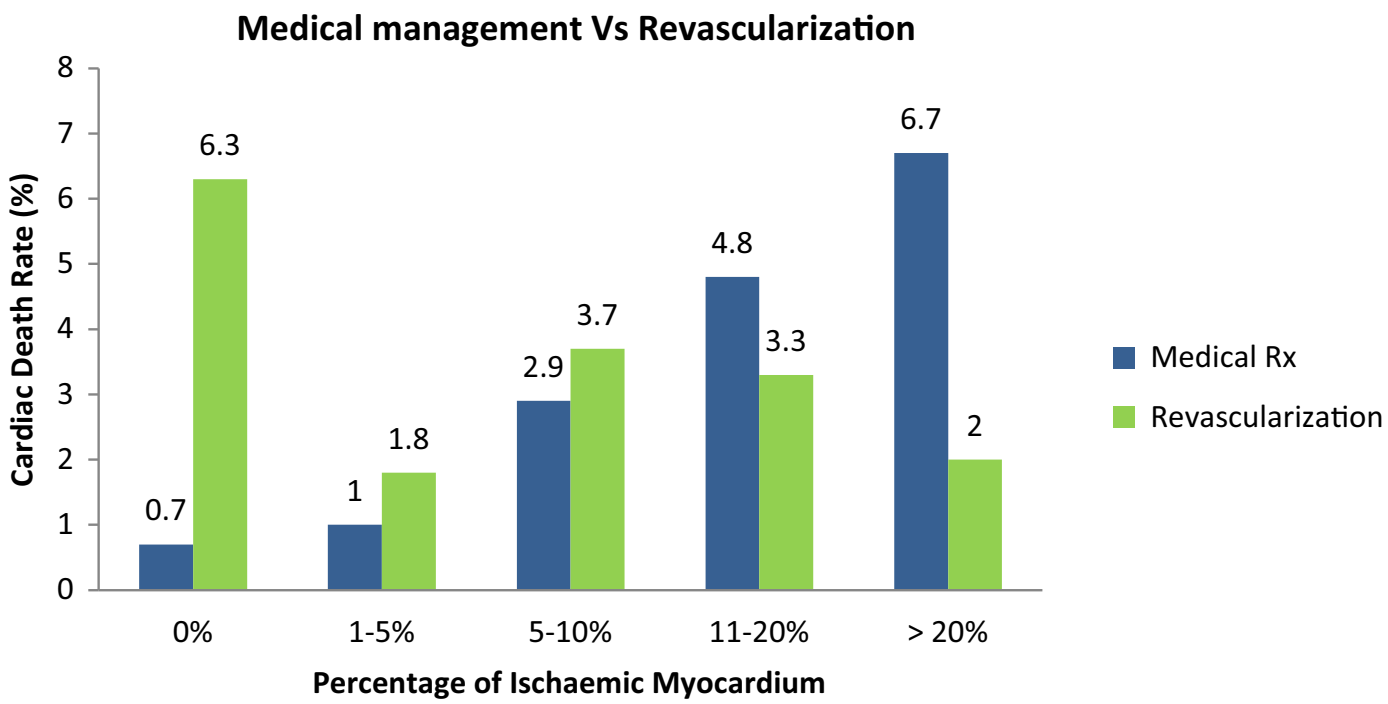

Figure 1. Cardiac death rates in patients with medical management and revascularization as a function of percentage of ischaemic myocardium.

revascularization. The study by $\mathrm{Li}$ et al reiterates the ideal of utilizing ischaemia to guide coronary revascularization. Does any ischaemia on non-invasive testing warrant revascularization? Hachamovitch et $\mathrm{al}^{8}$ performed an elegant study to try and answer that very question and concluded that when the degree of ischaemic burden is less than $10 \%$ of myocardium, the greatest benefit is with optimizing medical management. But when the ischaemic burden is greater than $10 \%$ of myocardium, the benefits of revascularization appear to outweigh the risks, and the prognostic benefit is also greater than that of medical therapy alone (Figure 1). Thus, it is essential that any report of a functional test used to evaluate the prevalence of ischaemia should quantify the ischaemic burden using a standardized left ventricular segmentation model.

Review of the literature on complete revascularization (CR) over incomplete revascularization (IR) shows a favorable trend toward CR but this appears to be mainly in the CABG studies. ${ }^{9}$ When CR and IR were directly compared based on PCI and CABG, there was no difference in mortality, non-fatal myocardial infarction (MI), or cerebro-vascular accidents between the two strategies, but higher repeat revascularization rates were noted in patients with higher SYNTAX scores. ${ }^{10,11}$ CR tends to be associated with favorable outcomes in those patients with complex, multi-vessel disease, particularly when surgery is the chosen revascularization modality. In the study by $\mathrm{Li}$ et al, prognosis was the poorest in those with IR according to both CA and MPI criteria, indicating more complex and severe disease that probably would have benefitted from CR with CABG instead of PCI. Li et al have also highlighted a very important point in groups 2 and 3 where CR is achieved based on CA parameters or MPI criteria but not both. There are considerable differences in defining CR and IR based on anatomical and functional data. This underpins one of the fundamental debates in cardiology. Although modern diagnostic imaging techniques have improved significantly from the early days of planar imaging or grainy $2 \mathrm{D}$ echocardiography, there still exists substantial false-positive and false-negative rates with these tests, particularly in cases of multi-vessel disease. In the case of MPI, multi-vessel disease can result in balanced perfusion or a perfusion defect in only one coronary artery territory. Assessment of perfusion in conjunction with wall motion abnormality and transient ischaemic dilatation does improve the accuracy of diagnosis of multi-vessel disease. ${ }^{12}$ In the case of stress echocardiography, the sensitivity of the technique to identify multi-vessel disease is only $50 \%$ when left ventricular function is normal. ${ }^{13}$

In recent years, Fractional flow reserve (FFR) has been shown to be able to identify ischaemia-inducing stenoses with a very high degree of accuracy. FFR is defined as the ratio of maximal blood flow to normal flow in an artery. FFR is 1 in a normal artery, whereas FFR $<0.75$ is indicative of a stenosis likely to cause down stream myocardial ischaemia with a sensitivity of $87 \%$ and specificity of $100 \% .{ }^{14}$ In the Fractional Flow Reserve vs Angiography for Multi-vessel Evaluation (FAME) study, ${ }^{15}$ the decision to deploy a stent was based on FFR measurements (FFR $<0.8$ ) or angiography alone. Significantly lesser stents were implanted in the FFR group as compared to the angiography group $(1.9 \pm 1.3$ vs $2.7 \pm 1.2, P<.001)$. At the end of 1 year, 
a composite end point comprising of death, non-fatal MI, and repeat revascularization was noted in $13.2 \%$ of the FFR group compared to $18.3 \%$ of the angiography group, $P=0.02$.

Measurement of fractional flow reserve and coronary flow reserve has been attempted with both CT and SPECT with some promising results. ${ }^{16-18}$ A detailed anatomical and physiological model of the myocardium and the coronary tree is created using the data from the CT coronary angiography, and principles of computational fluid dynamics are applied to these models to calculate pressure differences across coronary lesion. Dynamic tomographic scintigraphy acquisition has been problematic with conventional gamma cameras due to their inability to obtain sufficient counts rapidly. Some of the cameras using solid-state detectors appear to address some of these issues. Despite the initial successes, both these techniques are still very much in their infancy and we eagerly await data from larger trials.

FFR utilization appears to bridge the previously seemingly insurmountable gap between the anatomical information obtained by angiography and the functional information provided by the various non-invasive tests. It is increasingly clear that the decision to revascularize should be based on a combination of patient's detailed clinical assessment along with a risk score once the coronary anatomy has been studied. In addition, quantification of myocardial ischaemic burden using any one of the multitude of non-invasive diagnostic tests available would greatly beneficial. And finally judicious utilization of FFR measurement during angiography should ultimately help guide the clinician to choose the optimal strategy for the individual. Whether FFR bridges the gap created by the various definitions of CR and IR based on CA or MPI criteria remains to seen.

\section{References}

1. Keeley EC, Boura JA, Grines CL. Primary angioplasty versus intravenous thrombolytic therapy for acute myocardial infarction: A quantitative review of 23 randomised trials. Lancet 2003;361:13-20.

2. Boden WE, O'Rourke RA, Teo KK, COURAGE Trial Research Group, et al. Optimal medical therapy with or without PCI for stable coronary disease. N Engl J Med 2007;356:1503-16.

3. Jaarsma C, Leiner T, Bekkers SC, et al. Diagnostic performance of noninvasive myocardial perfusion imaging using single-photon emission computed tomography, cardiac magnetic resonance, and positron emission tomography imaging for the detection of obstructive coronary artery disease: A meta-analysis. J Am Coll Cardiol 2012;59:1719-28.
4. Mohr FW, Morice MC, Kappetein AP, et al. Coronary artery bypass graft surgery versus percutaneous coronary intervention in patients with three-vessel disease and left main coronary disease: 5-year follow-up of the randomised, clinical SYNTAX trial. Lancet 2013;381(9867):629-38.

5. Windecker S, Kolh P, Alfonso F, Collet JP, Cremer J, Falk V, et al. 2014 ESC/EACTS Guidelines on myocardial revascularization. Eur Heart J 2014;35:2541-619.

6. Jeremias A, Kaul S, Rosengart TK, et al. The impact of revascularization on mortality in patients with nonacute coronary artery disease. Am J Med 2009;122(2):152-61

7. Pepine CJ, Geller NL, Knatterud GL, et al. The Asymptomatic Cardiac Ischemia Pilot (ACIP) study: Design of a randomized clinical trial, baseline data and implications for a long-term outcome trial. J Am Coll Cardiol 1994;24:1-10.

8. Hachamovitch R, Hayes SW, Friedman JD, et al. Comparison of the short-term survival benefit associated with revascularization compared with medical therapy in patients with no prior coronary artery disease undergoing stress myocardial perfusion single photon emission computed tomography. Circulation 2003;107:2900-7.

9. Goss1 M, Faxon DP, Bell MR, et al. Complete versus incomplete revascularization with coronary artery bypass graft or percutaneous intervention in stable coronary artery disease. Circulation 2012;5:597-604.

10. Serruys PW, Unger F, Sousa JE, Arterial Revascularization Therapies Study Group, et al. Comparison of coronary-artery bypass surgery and stenting for the treatment of multivessel disease. N Engl J Med 2001;344:1117-24.

11. Sarno G, Garg S, Onuma Y, et al. ARTS-II investigators. Impact of completeness of revascularization on the 5-year outcome in percutaneous coronary intervention and coronary artery bypass graft patients (from the ARTS-II study). Am J Cardiol 2010;106:1369-75.

12. Lima RSL, Watson DD, Goode AR, et al. Incremental value of combined perfusion and function over perfusion alone by gated SPECT myocardial perfusion imaging for detection of severe three-vessel coronary artery disease. J Am Coll Cardiol 2003;42:64-70.

13. Marwick TH. Stress echocardiography. Heart 2003;89:113-8.

14. De Bruyne B, Pijls NH, Bartunek J, et al. Fractional flow reserve in patients with prior myocardial infarction. Circulation 2001; 104:157-62.

15. Tonino PA, De Bruyne B, Pijls NH, FAME Study Investigators, et al. Fractional flow reserve versus angiography for guiding percutaneous coronary intervention. N Engl J Med 2009;360:21324.

16. Koo BK, Erglis A, Doh JH, et al. Diagnosis of ischemia-causing coronary stenoses by noninvasive fractional flow reserve computed from coronary computed tomographic angiograms: Results from the prospective multicenter DISCOVER-FLOW (diagnosis of ischemia-causing stenoses obtained via noninvasive fractional flow reserve) study. J Am Coll Cardiol 2011;58:1989-97.

17. Jk Min, Leipsic J, Pencina MJ, et al. Diagnostic accuracy of fractional flow reserve from anatomic CT angiography. JAMA 2012;308:1237-45.

18. Ben-Haim S, Murthy VL, Breault C, et al. Quantification of myocardial perfusion reserve using dynamic SPECT imaging in humans: A feasibility study. J Nucl Med 2013;54:873-9. 